Discussion Paper 119

Institute for Empirical Macroeconomics

Federal Reserve Bank of Minneapolis

250 Marquette Avenue

Minneapolis, Minnesota 55480-0291

July 1997

\title{
International Portfolio Diversification and Labor/Leisure Choice*
}

\author{
Urban J. Jermann \\ The Wharton School, \\ University of Pennsylvania
}

\begin{abstract}
When marginal utility of consumption depends on leisure, investors will take this into account when allocating their wealth among different assets. This paper presents a multi-country general equilibrium model driven by productivity shocks, where labor-leisure and consumption are chosen endogenously. We use this framework to study the effect of leisure for optimal international diversification. We find that in the symmetric case the model's ability to help explain home-bias depends crucially on the level of substitutability between consumption and leisure.
\end{abstract}

*The views expressed herein are those of the author and not necessarily those of the Federal Reserve Bank of Minneapolis or the Federal Reserve System. 


\title{
International Portfolio Diversification and Labor/Leisure Choice
}

\author{
Urban J. Jermann \\ The Wharton School, University of Pennsylvania
}

July, 1997

\begin{abstract}
When marginal utility of consumption depends on leisure, investors will take this into account when allocating their wealth among different assets. This paper presents a multi-country general equilibrium model driven by productivity shocks, where labor-leisure and consumption are chosen endogenously. We use this framework to study the effect of leisure for optimal international diversification. We find that in the symmetric case the model's ability to help explain home-bias depends crucially on the level of substitutability between consumption and leisure.
\end{abstract}

\section{Introduction}

Investors in most countries have access to a large set of international financial instruments, however, they choose to invest mostly in domestic assets. ${ }^{1}$ With portfolio theory suggesting international diversification, we have one of the most robust puzzles in international finance. Explanations of this puzzle have been of two types. The first type of explanations suggests that frictions in international financial markets make foreign assets sufficiently less attractive to justify the foregone diversification benefits. Whereas the second type of explanations builds on frictions outside the financial markets to justify observed portfolio holdings as an optimal risk management strategy.

\footnotetext{
${ }^{1}$ For instance, French and Poterba (1991) document this issue.
} 
None of the available explanations seem entirely satisfactory to date, on the contrary, the puzzle may be even worse than what textbooks usually suggest. Indeed, Baxter and Jermann (1997) show that when one considers the large and nontradable human capital component of wealth, agents should hold even less domestic assets. The reason is that with domestic asset returns highly correlated with domestic marketable assets, hedging the nontradable human capital requires a reduction in the holdings of these domestic marketable assets. The present paper goes one step further and endogenizes the labor/leisure choice, with the objective of evaluating international diversification when preferences of consumption and leisure are nonseparable.

We present a multicountry model, where countries are subject to country specific productivity shocks. Households decide how much time to spend working in the market and chose portfolios that give them the necessary income to finance their consumption. In order to solve the portfolio problem analytically we adapt a method presented in Baxter, Jermann and King (1994). The complicated problem becomes tractable by working with linear approximations of the first order conditions, a technique widely used for solving macroeconomic models. Pareto efficient consumption allocations can then be supported in a decentralized equilibrium by appropriately chosen international portfolios. ${ }^{2}$

In the symmetric case, where all countries are ex-ante identical, we solve for the agents' portfolios as a function of preference and technology parameters. The solution shows that, in order for the considered nonseparability to help explain home-bias, consumption and leisure need to be substitutes. This is qualitatively consistent with findings from the household production literature, for instance Greenwood, Rogerson and Wright (1995), that suggests substitutability between market produced consumption and goods and services produced with non-market time. In this case, when agents work a lot, they have little time for non-market production (cooking, cleaning, child-care etc.) and thus they value market consumption a lot-by holding domestic claims they will get the necessary purchasing power to finance increased market consumption.

In the remainder of this paper, we will first characterize Pareto efficient allocations. Second, we derive the portfolio holdings required to support

\footnotetext{
${ }^{2}$ Leung (1995) solves a 2 country model with preferences that are nonseparable in consumption and leisure. For his case, the restrictions on preferences, required to obtain a closed form solution, rule out the possibility that the nonseparability can help explain home-bias.
} 
optimal allocations. And third, we solve the symmetric case.

\section{Optimal allocations}

This section develops a multi-country general equilibrium model that we will use to study the determinants of optimal portfolio choice in the presence of leisure. Our strategy is to first characterize optimal consumption allocations in this section. Then, the second step is to determine the portfolios that will support these optimal allocations, which we undertake in the next section of the paper.

We have a $J$-country economy, that is atemporal and that is subject to country-specific productivity shocks. Each country representative consumes $c_{j}$ of the consumption good, works a fraction $n_{j}$ of his non-sleeping time and gets $l_{j}=1-n_{j}$ of leisure. Pareto optimal allocations are determined by maximizing a weighted sum of individual country utilities $v_{j}\left(c_{j}, 1-n_{j}\right)$; letting $\omega_{j}$ denote the weight given to country $j$ with $\sum_{j=1}^{J} \omega_{j}=1$, this weighted sum is given by:

$$
\sum_{j=1}^{J} \omega_{j} v_{j}\left(c_{j}, 1-n_{j}\right) .
$$

The world resource constraint for the consumption good is given by:

$$
\sum_{j=1}^{J} \pi_{j} c_{j}=\sum_{j=1}^{J} \pi_{j} y_{j}=\sum_{j=1}^{J} \pi_{j} A_{j} f_{j}\left(n_{j}\right) \equiv Y
$$

where $A_{j}$ is productivity, $y_{j}$ is country $j$ 's output and $Y$ is world output. The resource weights $\pi_{j}$ allow countries to vary in terms of economic size.

The first-order conditions describing optimal consumption allocations are

$$
\omega_{j} \frac{\partial v_{j}\left(c_{j}, 1-n_{j}\right)}{\partial c_{j}}=\lambda \pi_{j}, \quad j=1,2, \ldots, J
$$

where $\lambda$ is the multiplier on the world resource constraint. The first-order conditions describing optimal labor-leisure allocation are:

$$
\omega_{j} \frac{\partial v_{j}\left(c_{j}, 1-n_{j}\right)}{\partial n_{j}}=-\lambda \pi_{j} A_{j} \frac{\partial f_{j}\left(n_{j}\right)}{\partial n_{j}}, \quad j=1,2, \ldots, J .
$$

These conditions are standard and imply that marginal utility of consumption is equalized across agents and, for the latter, that for each agent the marginal product of labor equals its marginal cost. 


\subsection{Properties of optimal consumption allocations}

We now proceed to determine the properties of optimal consumption allocations. Following the method in Baxter, Jermann and King (1995) we differentiate first order conditions and we interpret terms such as $d c$ as small endogenous deviations from the deterministic solution brought about by small shocks to the country specific productivity levels $d A$. We leave it open how small exactly these shocks are. A large literature on the accuracy of loglinear approximations suggests such methods to be extremely precise for models calibrated to business cycle fluctuations, see for instance Taylor and Uhlig (1990)..$^{3}$

Totally differentiating equation (2), we find that

$$
\frac{d c}{c}=\left[\frac{1}{\xi_{c c}}\right] \frac{d \lambda}{\lambda}+\left[\frac{\xi_{c l} \cdot n}{\xi_{c c}(1-n)}\right] \frac{d n}{n}
$$

where we do not include country subscripts since we are describing general properties. Parameters like $\xi_{x_{1}, x_{2}}$ stand for the elasticity of the marginal utility of $x_{1}$ with respect to $x_{2}$, formally, $\xi_{x_{1}, x_{2}}=\frac{\partial^{2} v(.)}{\partial x_{1} \partial x_{2}} \cdot \frac{x_{2}}{\partial v(.)}$. We further label parameters as:

$$
\begin{gathered}
\eta^{\lambda} \equiv\left[\frac{1}{\xi_{c c}}\right], \text { and } \\
\eta^{n} \equiv\left[\frac{\xi_{c l} \cdot n}{\xi_{c c}(1-n)}\right] .
\end{gathered}
$$

Equation (4) tells us whether an increase in $n$ raises or lowers the optimal level of $c$, holding fixed the shadow price for world output $\lambda$. If consumption and leisure are complements, i.e., $\xi_{c l}>0$, then marginal utility of $c$ decreases with $n$ and it is optimal to allocate larger quantities of the consumption good to countries that work less. Conversely, if consumption and leisure are substitutes, that is when $\xi_{c l}<0$, so that the marginal utility of $c$ increases

\footnotetext{
${ }^{3}$ Although the preference specification of the model here is isomorphic to the model with nontraded goods in Baxter, Jermann and King (1995), the model's structure is fundamentally different. In the model here, leisure is endogenously determined jointly with endogenous output. In the model with nontraded goods, the outputs of the traded and nontraded good sectors are exogenous endowments and consumption of the nontraded good equals the exogenous output.
} 
with increases of $n$, then the optimal allocation involves lower allocations of the consumption good to countries working relatively more.

In our analysis below, we will allow the elasticities $\eta^{\lambda}$ and $\eta^{n}$ to differ by country, where countries are distinguished by the subscript $j=1,2, \ldots, J$. These elasticities may differ across countries because the ratio $\pi / \omega$ differs across countries or because the benchmark level of $n$ at which the elasticities are evaluated differs across countries. ${ }^{4}$

An additional requirement for the Pareto optimal allocation is resourcefeasibility. Totally differentiating the resource constraint (1) we obtain the following:

$$
\frac{d Y}{Y}=\sum_{j=1}^{J} \theta_{j} \eta_{j}^{\lambda} \frac{d \lambda}{\lambda}+\sum_{j=1}^{J} \theta_{j} \eta_{j}^{n} \frac{d n_{j}}{n_{j}}=\sum_{j=1}^{J} \theta_{j} \eta_{j}^{\lambda} \frac{d \lambda}{\lambda}+\eta^{N} \frac{d N}{N},
$$

where $\theta_{j}=\pi_{j} c_{j} / Y$ and the latter equality follows from the definitions $\eta^{N} \equiv$ $\sum_{j=1}^{J} \theta_{j} \eta_{j}^{n}$ and $d N / N=\left[\sum_{j=1}^{J} \theta_{j} \eta_{j}^{n} \frac{d n_{j}}{n_{j}}\right] / \eta^{N}$. This last equation determines the effect on $\lambda$ of changes in output and labor supply in the world economy. Using this result together with equation (4), we find that, for country $j$,

$$
\frac{d c_{j}}{c_{j}}=\frac{\eta_{i}^{\lambda}}{\sum_{j=1}^{J} \theta_{j} \eta_{j}^{\lambda}} \frac{d Y}{Y}-\frac{\eta_{i}^{\lambda}}{\sum_{j=1}^{J} \theta_{j} \eta_{j}^{\lambda}} \eta^{N} \frac{d N}{N}+\eta_{j}^{n} \frac{d n_{j}}{n_{j}},
$$

where the first two terms are the effects of aggregate displacements operating through $\lambda$.

This pair of expressions reveals the core economics of our multi-country model. From equation (5) we have the intuitive result that the world marginal utility of $Y$ falls with increases in $Y$ since $\sum_{j=1}^{J} \theta_{j} \eta_{j}^{\lambda}<0$. Further, the effect on $\lambda$ of an increase in $n_{j}$ depends on the sign of $\eta_{j}^{n}$ and on the importance of country $j$ in the world economy, as reflected in $\theta_{j}$. Further, we can view $\lambda$ as influenced by a world supply shock to the output of the consumption good, $d Y / Y$, and a world demand shock $d N / N$. From equation (6), we see that there is a positive effect of the world supply shock $d Y / Y$ on the consumption allocation of country $j$. Further, there are also effects of the country's work effort $\left(d n_{i} / n_{i}\right)$ and the work effort of the whole world $(d N / N)$, whose directions depend on preference parameters we discuss later in the paper.

\footnotetext{
${ }^{4}$ Obviously, neither the shadow price, $\lambda$, nor labor supply, $n$, are exogenous in this model. But, interpreting the consumption allocation in this particular way will show to be useful for building intuition and for solving the model.
} 
If the various countries have equal elasticities $\eta_{i}^{n}=\eta^{n}=\eta^{N}$, and $\eta_{i}^{\lambda}=\eta^{\lambda}$, then we have the following version of (6):

$$
\frac{d c_{i}}{c_{i}}=\frac{d Y}{Y}+\eta^{n}\left(\frac{d n_{i}}{n_{i}}-\frac{d N}{N}\right) .
$$

where $d N / N \equiv \sum_{j=1}^{J} \theta_{j}\left(d n_{j} / n_{j}\right)$.

In (7), changes in world supplies of $Y$ are shared equally if $\eta^{n}=0$, which would be the case for separable utility. If $\eta^{n} \neq 0$, then an additional reallocation of the consumption good is undertaken based on an individual country's work effort relative to the world average. Thinking about leisure as producing shifts in the demand for the consumption good, equation (7) is very intuitive: changes in world demand for $c$ must be frustrated by adjustments in its shadow price $(\lambda)$ since there is a given stock to be allocated. It is only if there is a relative demand shock that a country's allocation is affected.

In the preceding section we provided a characterization of optimal consumption allocations in a general equilibrium, multi-country production model with stochastic productivity. The optimal allocations were derived under the assumption that a benevolent social planner maximized a weighted sum of individual country utilities. In practice, of course, individual consumption opportunities are not determined by a social planner, but instead are the result of individual choices concerning the decision to invest, consume and work. Thus our next task is to characterize portfolios that can support the optimal allocations. Recall that the optimal allocation of the consumption good for an individual country depended on world output, the country's labor supply and the world labor supply. That is: equation (6) was of the form

$$
d c_{j}=\beta_{j}^{Y} d Y+\beta_{j}^{N} d N+\beta_{j}^{n} d n_{j} .
$$

Viewing $d Y, d N$, and $d n_{j}$ as three sources of uncertainty for country $j$ 's consumption, equation (8) shows how optimal consumption - and therefore optimal portfolio returns in the decentralized equilibrium - must respond to the various sources of uncertainty or risk factors.

\section{$3 \quad$ Asset and portfolio payoffs}

We define an "equity claim" in this atemporal world to be a claim to a dividend, given by output minus labor income. Restricting the production 
function to be of the form $f_{j}\left(n_{j}\right)=n_{j}^{\alpha_{j}}$, the dividend, which is the income that goes to the fixed factor is then simply equal to $\left(1-\alpha_{j}\right) y_{j}$. Individual consumers will choose portfolios of such equity claims to all countries' outputs. As we are about to show, countries' asset holding comprise quantities of three different mutual funds, a world equity fund, the country's domestic equity fund and a world hedge fund for labor supply shocks. We will here first define these funds and show how their returns depend on the three sources of uncertainty considered for the individual consumption choice, namely $d Y$, $d N$, and $d n_{j}$. Second, we will solve for the optimal quantities of each fund.

First, the world equity fund pays the following return:

$$
d \mathbf{E}=\sum_{j=1}^{J} w_{j} d \mathbf{e}_{j}=\sum_{j=1}^{J} w_{j}\left(1-\alpha_{j}\right) d y_{j} .
$$

Ideally, we would like the return to this world equity fund, to depend only on the world output of the consumption good, i.e., we would like $d \mathbf{E}$ to depend only on $d Y$. This can be accomplished by choosing the portfolio weights $w_{j}$ to satisfy

$$
w_{j}=W \frac{\pi_{j}}{\left(1-\alpha_{j}\right)}
$$

so that $\sum_{j=1}^{J} w_{j}\left(1-\alpha_{j}\right) d y_{j}=W d Y$. Further, requiring that $\sum_{j=1}^{J} w_{j}=$ 1 determines the constant $W=\left[\sum_{j=1}^{J} \pi_{j} /\left(1-\alpha_{j}\right)\right]^{-1}$. With the portfolio weights determined in this way, we may write:

$$
d \mathbf{E}=\Lambda_{E} d Y .
$$

Second, the domestic equity fund returns:

$$
d \mathbf{e}_{j}=\left(1-\alpha_{j}\right) d y_{j}=\left(1-\alpha_{j}\right)\left[\alpha\left(y_{j} / n_{j}\right) d n_{j}+\left(y_{j} / A_{j}\right) d A_{j}\right] .
$$

Normally, we would solve this equation by substituting out the endogenous variable in the right hand side, $d n_{j}$, but for our purpose of solving for portfolio shares we will substitute out the productivity shock, $d A_{j}$. To do this, we use the linearized version of the first order condition for labor supply, 3 , to substitute out $d A_{j}$, we then use 6 and 5 , to get rid of $d \lambda / \lambda$ and $d c_{j} / c_{j}$. The domestic equity return is then found to depend on all three risk factors:

$$
d \mathbf{e}_{j}=\Lambda_{j}^{Y} d Y+\Lambda_{j}^{N} d N+\Lambda_{j}^{n} d n_{j}
$$


with the $\Lambda$ coefficients being functions of the different demand elasticities, production elasticities and country weights.

Finally, we need to define the world hedge fund for labor supply shocks:

$$
d \mathbf{H}=\sum_{j=1}^{J} w_{j}^{H} d \mathbf{e}_{j}=\sum_{j=1}^{J} w_{j}^{H}\left(\Lambda_{j}^{Y} d Y+\Lambda_{j}^{N} d N+\Lambda_{j}^{n} d n_{j}\right) .
$$

We want this fund to be useful in hedging the risk associated with world demand shocks on consumption coming from changes in the world labor supply. We therefore require that its return does depend only on the world risk factors, $d Y$ and $d N$, but not on any $d n_{j}$ directly. This can be accomplished by choosing the portfolio weights $w_{j}^{H}$ to satisfy

$$
w_{j}^{H} \Lambda_{j}^{n}=W^{H} N \frac{\theta_{j} \eta_{j}^{n}}{n_{j} \eta^{N}}
$$

so that $\sum_{j=1}^{J}\left(w_{j}^{H} \Lambda_{j}^{n}\right) d n_{j}=W^{H}(d N)$. Further, requiring that $\sum_{j=1}^{J} w_{j}^{H}=$ 1 determines the constant $W^{H}=\left[\sum_{j=1}^{J}\left(N \theta_{j} \eta_{j}^{n} /\left(\eta^{N} n_{j} \Lambda_{j}^{n}\right)\right)\right]^{-1}$. With the portfolio weights determined in this way, we may write

$$
d \mathbf{H}=\sum_{j=1}^{J}\left(w_{j}^{H} \Lambda_{j}^{Y}\right) d Y+\left(\sum_{j=1}^{J}\left(w_{j}^{H} \Lambda_{j}^{N}\right)+W^{H}\right) d N,
$$

which is a payoff structure of the form:

$$
d \mathbf{H}=\Lambda_{H}^{Y} d Y+\Lambda_{H}^{N} d N .
$$

\section{Supporting optimal consumption}

In order to purchase his optimal allocation, an individual living in country $j$ must have purchasing power equal to $c_{j}$. The displacement in expenditure arising from displacements in world and national risk factors is given by

$$
d c_{j}=\beta_{j}^{Y} d Y+\beta_{j}^{N} d N+\beta_{j}^{n} d n_{j} .
$$

This expression suggests that one way to generate the income necessary to buy the optimal allocation is to hold appropriate quantities of three assets: the asset representing a claim on the world output, $d Y$; the asset having a 
payout representing the world labor supply, $d N$; and the asset having a payout representing the individual's home country's labor supply, $d n_{j}$. However, these assets are not directly available to the investor, instead he will replicate their payouts by holding a portfolio of the three mutual funds defined above. A little algebra shows that the risk factors are replicated by the following combinations of mutual funds:

$$
\begin{gathered}
d Y=\left(\frac{1}{\Lambda_{E}}\right) d \mathbf{E} \\
d N=\left(-\frac{\Lambda_{H}^{Y}}{\Lambda_{E} \Lambda_{H}^{N}}\right) d \mathbf{E}+\left(\frac{1}{\Lambda_{H}^{N}}\right) d \mathbf{H} \\
d n_{j}=\left(\frac{1}{\Lambda_{E} \Lambda_{j}^{n}}\right)\left(\frac{\Lambda_{j}^{N} \Lambda_{H}^{Y}}{\Lambda_{H}^{N}}-\Lambda_{j}^{Y}\right) d \mathbf{E}-\left(\frac{\Lambda_{j}^{N}}{\Lambda_{H}^{N} \Lambda_{j}^{n}}\right) d \mathbf{H}+\left(\frac{1}{\Lambda_{j}^{n}}\right) d \mathbf{e}_{i} .
\end{gathered}
$$

Having determined how to construct the risk factors as linear combinations of the mutual funds, it is straightforward to characterize the quantities of each of these funds that an individual must hold in order to be able to purchase his optimal consumption basket. Substituting for $d Y, d N$, and $d n_{i}$ from (12)-(14), we have:

$$
d c_{i}=\vartheta_{i}^{E} d \mathbf{E}+\vartheta_{i}^{H} d \mathbf{H}+\vartheta_{i}^{e} d \mathbf{e}_{i}
$$

where the coefficients - and thus the holdings - for each of the mutual funds are:

$$
\begin{gathered}
\vartheta_{j}^{E}=\frac{1}{\Lambda_{E}} \beta_{j}^{Y}-\left(\frac{\Lambda_{H}^{Y}}{\Lambda_{E} \Lambda_{H}^{N}}\right) \beta_{j}^{N}-\left(\frac{1}{\Lambda_{E} \Lambda_{j}^{n}}\right)\left(\Lambda_{j}^{E}-\frac{\Lambda_{i}^{N} \Lambda_{H}^{E}}{\Lambda_{H}^{N}}\right) \beta_{j}^{n} \\
\vartheta_{j}^{H}=\left(\frac{1}{\Lambda_{H}^{N}}\right) \beta_{j}^{N}-\left(\frac{\Lambda_{i}^{N}}{\Lambda_{H}^{N} \Lambda_{i}^{n}}\right) \beta_{j}^{n} \\
\vartheta_{j}^{e}=\left(\frac{1}{\Lambda_{j}^{n}}\right) \beta_{j}^{n} .
\end{gathered}
$$




\subsection{Nontradable human capital}

We have sofar abstracted from the fact that claims to labor income are nontradable. That is, we have implicitly assumed that labor income can be traded directly. In fact, if we assume that labor income cannot be traded directly, agents will use tradable securities to hedge away labor income risk by selling a portfolio that replicates labor income. For our particular production function, $y_{j}=A_{j} n_{j}^{\alpha_{j}}$, the replicating portfolio is straightforward to determine. Indeed, given that returns to labor and the fixed factor are just fixed fractions of the returns to total output, $d y_{j}$, the return on human capital, $\alpha_{j} d y_{j}$, is thus just a linear function of the domestic equity return:

$$
\alpha_{i} d y_{i}=\sum_{j=1}^{J} w_{j}^{h c} d \mathbf{e}_{j}=\frac{\alpha_{i}}{1-\alpha_{i}} d \mathbf{e}_{i} .
$$

Therefore, the overall portfolio holdings computed above have to be adjusted by the additional hedge for labor income of

$$
-\frac{\alpha_{i}}{1-\alpha_{i}} d \mathbf{e}_{i}
$$

\section{The symmetric case}

This section presents results for a world economy in which all countries are identical in terms of initial conditions, although they are subject to different productivity shocks. That is: we assume the following initial conditions: $c_{i}=c=y=Y, n_{i}=n$ and $\pi_{i}=1 / J$. With identical preferences across countries, the central elasticities are the same across countries: $\eta_{i}^{\lambda}=\eta^{\lambda}$, $\eta_{i}^{n}=\eta^{n}=\eta^{N}$. To solve for the optimal portfolio allocation we can now simply follow the steps outlined for the general case.

First, it is straightforward to see that both world funds are identical, so that:

$$
d \mathbf{E}=d \mathbf{H}=\frac{1}{J} \sum_{j=1}^{J} d \mathbf{e}_{j} ;
$$

that is, the world funds are just equally weighted, with units equal to one country's size share. After somewhat tedious but straightforward algebrawhose details are available from the author - portfolio weights simplify significantly to:

$$
\vartheta^{E}=\frac{1}{1-\alpha}
$$




$$
\begin{gathered}
\overline{\vartheta^{e}}=\vartheta^{e}-\frac{\alpha}{1-\alpha}=\frac{\beta^{n}}{\Lambda^{n}}-\frac{\alpha}{1-\alpha} \\
\vartheta^{H}=-\vartheta^{e} .
\end{gathered}
$$

Where $\vartheta^{e}$ is a function of the underlying parameters of the model. Clearly, the optimal portfolio is simply a weighted portfolio of a fully diversified world fund and the domestic fund. Let us look now in detail at $\vartheta^{e}$.

\subsection{Separable utility}

To gain intuition about the solution let us first assume that utility of consumption and leisure is separable. This implies that $\xi_{c l}=0$, and that $\vartheta^{e}=0$, so that:

$$
\begin{gathered}
\vartheta^{E}=\frac{1}{1-\alpha} \\
\overline{\vartheta^{e}}=-\frac{\alpha}{1-\alpha} \\
\vartheta^{H}=0 .
\end{gathered}
$$

Here, the investor has a short position in the domestic equity fund, de, that is only partially offset by domestic holdings in the world fund, $d \mathbf{E}$. In particular, if the country represents a share $\pi$ of the world market, then it holds $\frac{\pi}{1-\alpha}$ of its wealth in domestic equity through the world fund but $-\frac{\alpha}{1-\alpha}$ through the domestic fund. Therefore, with $\pi-\alpha<0$ for all countries, that is, the country's share in the world market is smaller than the labor share, the domestic holdings are negative. This is the result of Baxter and Jermann (1997), suggesting that the diversification puzzle is worse than you may have thought. 


\subsection{Nonseparable utility}

The case for which $\xi_{c l} \neq 0$ gives us

$$
\begin{aligned}
\vartheta^{e}= & \frac{\beta^{n}}{\Lambda^{n}}=\frac{\eta^{n}}{(1-\alpha)\left(1-\frac{\xi_{l l} \xi_{c c}-\xi_{l c} \xi_{c l}}{\xi_{c c}} \frac{n}{1-n}\right)}= \\
& \frac{\frac{\xi_{c l}}{\xi_{c c}} \frac{n}{1-n}}{(1-\alpha)\left(1-\frac{\xi_{l l} \xi_{c c}-\xi_{l c} \xi_{c l}}{\xi_{c c}} \frac{n}{1-n}\right)} \\
= & \frac{\frac{\xi_{c l}}{\xi_{c c}} \frac{n}{1-n}}{(1-\alpha)\left(1+\frac{1}{\varepsilon_{n, w}}\right)},
\end{aligned}
$$

where $\varepsilon_{n, w}$ is the (compensated) elasticity of labor supply with respect to the wage rate. The denominator being always positive because of concavity, the sign of $\vartheta^{e}$ depends on the sign of $\xi_{c l}$, given that $\xi_{c c}<0$ to insure concavity. ${ }^{5}$ With $\xi_{c l}<0$, consumption and leisure are substitutes, and $\vartheta^{e}>0$. This finding is indeed very intuitive, with consumption and leisure substitutes, consumption is highly valued in periods when work effort is high. On the other hand, these are the periods when work effort creates a positive effect on domestic output. Therefore, a domestic claim provides the right hedge. This story seems to fit well with a somewhat broader notion of leisure as time that is used to privately produce goods and services, the way it is suggested by the literature on household production, for instance by Greenwood, Rogerson and Wright (1995). Clearly, when working long-hours for market activity, little time is available for cooking, cleaning, child-caring etc. and these goods and services need now to be purchased in the market. By holding domestic claims that have high payouts agents have the ability to afford these goods and services.

The effect of the labor supply elasticity also seems to fit well with our intuition. For instance, with a very small labor supply elasticity, domestic labor supply does not move much, and there is not much need for hedging this risk factor for consumption.

\footnotetext{
${ }^{5}$ Concavity requires that $\xi_{c c}$ and $\xi_{l l}$ are both negative, whereas $\xi_{c l}$ and $\xi_{l c}$ may be either positive or negative as long as $\xi_{c c} \xi_{l l}-\xi_{c l} \xi_{l c}>0$ for overall concavity. Given that $\xi_{c l}$ and $\xi_{l c}$ take their sign from the cross derivative, they both have the same sign.
} 


\subsection{A quantitative look}

We look here at the issue of whether the effect of nonseparable leisure can plausibly explain home-bias, also considering that the nontraded labor income puts us in a very unfavorable starting position. We look at this issue by using two possible ways of parameterizing preferences. Overall, it appears that a substantial degree of substitutability is required to explain large home-bias.

To get a better grip on the preference parameters we first restrict preferences to have the following form:

$$
v(c, 1-n)=c^{1-\gamma} /(1-\gamma) * \nu(1-n),
$$

that is, CRRA in consumption and multiplicatively separable in leisure. This specification is widely used in macroeconomics because it guarantees the existence of a balanced growth path, as shown in King, Plosser and Rebelo (1987). This restriction implies that $\xi_{c c}=-\gamma$ and that $\xi_{l c}=1-\gamma$, which requires that the sign of $(1-\gamma)$ is equal to the sign of $\xi_{c l}$, because $\xi_{l c}$ and $\xi_{c l}$ both take their sign from the same cross-derivative. So that

$$
\overline{\vartheta^{e}}=\vartheta^{e}-\frac{\alpha}{1-\alpha}=\frac{\frac{\xi_{c l}}{-\gamma} \frac{n}{1-n}}{(1-\alpha)\left(1+\frac{1}{\varepsilon_{n, w}}\right)}-\frac{\alpha}{1-\alpha},
$$

is positive if risk aversion, $\gamma$, is larger than 1 . It is clear, that by making $\xi_{c l}$ sufficiently large in absolute value, any portfolio share is consistent with the model. However, given the little available direct information about this parameter, we will also consider an alternative preference specification.

A second way of parameterizing preferences is:

$$
v(c, 1-n)=\frac{1}{1-\gamma} \varphi(c, 1-n)^{1-\gamma},
$$

with $\varphi(\cdot)$ being a constant return to scale aggregator. As is well known, the local behavior of these preferences can be described by only three parameters, risk aversion, $\gamma$, the inverse of the elasticity of substitution, $\mu$, and the value share for consumption $s_{c}$ (and thus leisure $s_{l}=1-s_{c}$ ); that is, locally, this function is like a CES. ${ }^{6}$

\footnotetext{
${ }^{6}$ Value shares are defined as $s_{x}=\frac{\left(\frac{\partial \varphi()}{\partial x}\right) \cdot x}{\varphi()}$.
} 
After some algebra, the share of the domestic fund can be written as:

$$
\vartheta^{e}=\frac{\left[\frac{s_{l}(\gamma-\mu)}{s_{c} \gamma+s_{l} \mu}\right] \frac{n}{1-n}}{(1-\alpha)\left(1+\frac{1}{\varepsilon_{n, w}}\right)},
$$

with $\mu$ being the inverse of the elasticity of substitution - the extent to which individual dislike substitution across the two goods. ${ }^{7}$ However, $\varepsilon_{n, w}$ is here no longer a free parameter, but it depends on the other 3 coefficients. Solving further we can write that:

$$
\overline{\vartheta^{e}}=\vartheta^{e}-\frac{\alpha}{1-\alpha}=\frac{\left[\frac{s_{l}(\gamma-\mu)}{s_{c} \gamma+s_{l} \mu}\right] \frac{n}{1-n}}{(1-\alpha)\left(1+\left[\frac{\gamma \mu}{s_{c} \gamma+s_{l} \rho}\right] \frac{n}{1-n}\right)}-\frac{\alpha}{1-\alpha},
$$

where the sign of $\vartheta^{e}$ depends the sign of $\gamma-\mu$, which, as above, determines whether consumption and leisure are substitutes or complements.

To get some idea about quantitative implications let us calibrate this last expression with the following values: the fraction of non-sleeping time spent working $n=0.33$, labor share $\alpha=0.6$, risk aversion $\gamma=3 .{ }^{8}$ To a first approximation value shares may not be too different from the time fraction spent at the respective activities, so that $s_{c}=n$ and $s_{l}=1-n$. Figure 1 plots the holding of the domestic fund $\overline{\vartheta^{e}}$ as a function of $\mu$. In order to overcome the effect of the nontraded labor income and to bring the holding of the domestic funds above 0 an elasticity of substitution of $4,(\mu=.25)$, is required. Although not entirely inconsistent with estimates of this parameter in the household production literature, as for instance in Rupert, Rogerson and Wright (1996), such values are on the high side. ${ }^{9}$

\footnotetext{
${ }^{7}$ The definition of risk aversion, $\gamma$, is of course here different from the previous one.

${ }^{8}$ The Michigan time survey reports nonsleeping time spend working in the market at 0.33. The labor share is a standard value in the RBC literature.

${ }^{9}$ In addition to the holdings of the domestic equity funds, the two world funds also contain together $\pi\left(\frac{1}{1-\alpha}-\vartheta^{e}\right)$ in domestic claims.
} 


\section{References}

Baxter, Marianne and Jermann, Urban, J., 1997, "The international diversification puzzle is worse than you think," American Economic Review, March.

Baxter, Marianne and Jermann, Urban, J., 1996, "Household production and the excess sensitivity of consumption to current income, unpublished paper.

Baxter, Marianne, Jermann, Urban, J., and King, Robert, G., 1995, "Nontraded goods, nontraded factors, and international non-diversification," with M. Baxter and R. G. King, NBER Working Papers No. 5175.

French, Kenneth and Poterba, James, 1991, "Investor diversification and international equity markets," American Economic Review 81, 222-226.

Greenwood, Jeremy, Rogerson, Richard, and Wright, Randall, 1995, "Household production in real business cycle theory," in: Frontiers of Business Cycle Research, (T. Cooley, ed.), Princeton: Princeton University Press.

King, Robert, G., Plosser, Charles, I., and Rebelo Sergio, 1988, "Production, Growth and Business cycles," Journal of Monetary Economics, 195-232.

Leung, Charles, 1995, "Does non-traded input necessarily deepen the international non-diversification puzzle I?: The one-good case,"Economic Letters, 281-285.

Rupert, Peter, Rogerson, Richard, and Wright, Randall, 1996, "Homework in labor economics: Householdo production and intertemporal substitution," manuscript, Federal Reserve Bank of Cleveland.

Taylor, John, B. and Uhlig, Harald, 1990, "Solving nonlinear stochastic growth models: A comparison of alternative solution methods," Journal of Business \& Economics Statistics, v8n1, 1-17. 
Figure 1:

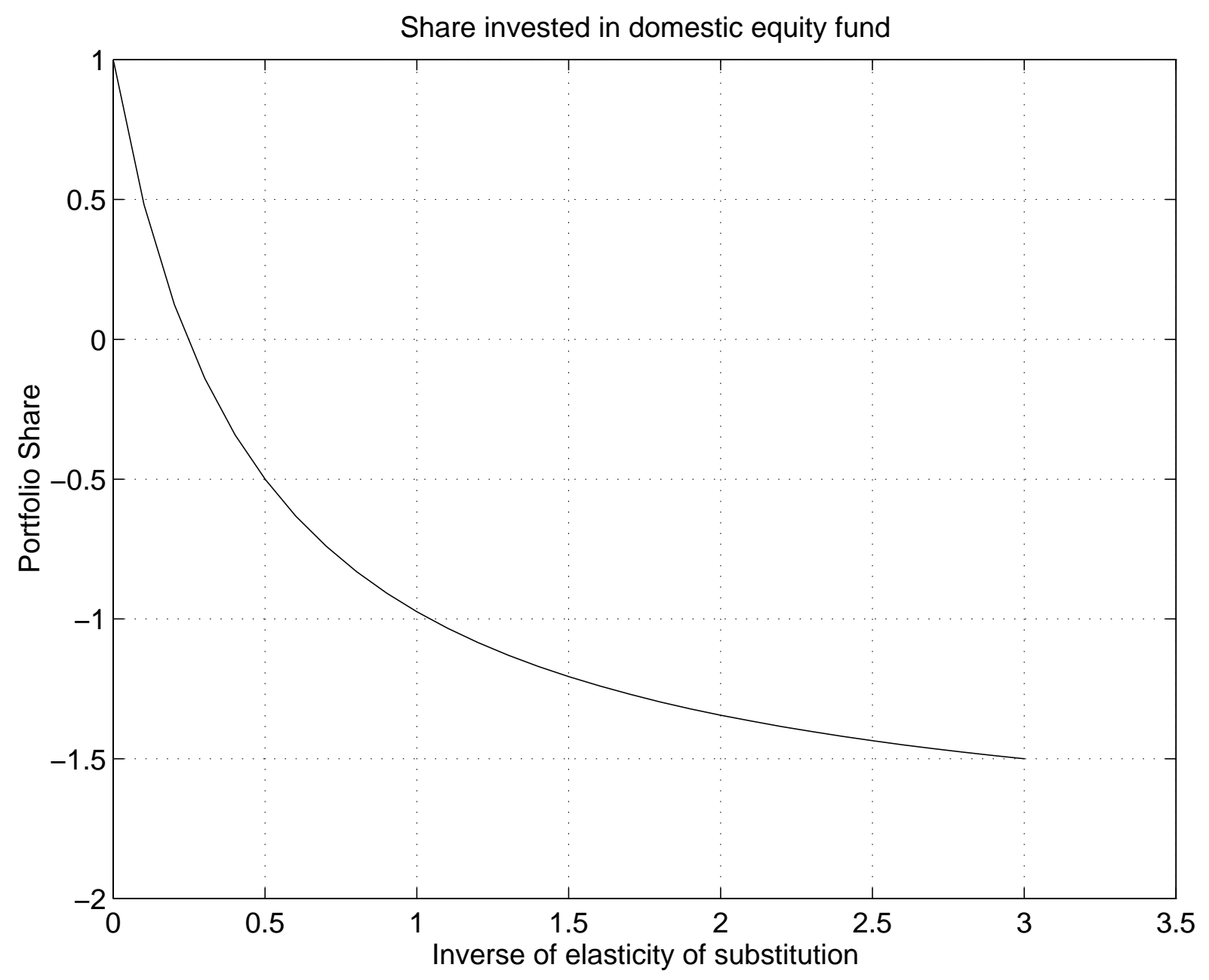

